\section{COUP-TFI: an intrinsic factor for early regionalization of the neocortex}

\author{
Cheng Zhou, Sophia Y. Tsai, and Ming-Jer Tsai ${ }^{1}$ \\ Department of Molecular and Cellular Biology, Program \\ in Developmental Biology, Baylor College of Medicine, \\ Houston, Texas 77030, USA
}

Regionalization of the cerebral cortex is thought to involve two phases: an early regionalization phase and a later refinement phase. It has been shown that early regionalization of the neocortex does not require thalamic inputs and is regulated by intrinsic factors. Recently, two such intrinsic factors, $\operatorname{Pax} 6$ and $E m x 2$, have been identified. In this study, we identified COUP-TFI as a regulatory factor for early neocortical regionalization. The spatial and temporal expression pattern of COUPTFI suggested a role in specification of the neocortex and in maintaining cortical identity. Altered region-specific expression of marker genes in the cortex as well as miswired area-specific connections between the cortex and the thalamus in COUP-TFI null mice indicate COUP-TFI plays a critical role in regulating early regionalization. Our results substantiate that COUP-TFI, an intrinsic factor, may work in concert with $\operatorname{Pax} 6$ and $E m x 2$ to specify neocortical identity.

Received May 22, 2001; revised version accepted June 29, 2001.

The mammalian cerebral cortex has a very sophisticated architecture. Tangentially, it consists of many discrete areas with distinct connections and cytoarchitecture. However, the mechanism(s) whereby the neocortex is specified and regionalized still are largely unknown. Two popular hypotheses have been proposed: the protomap hypothesis, which emphasizes genetic influences, proposes that cortical neurons are prespecified when they are born in the ventricular zone (Rakic 1988); in contrast, the protocortex hypothesis favors the role of epigenetic factors, mainly thalamocortical innervation, in cortical regionalization (O'Leary 1989). Transplantation studies have provided several lines of evidence supporting the protocortex hypothesis during postnatal development while other studies, some of which also involve transplantation experiments, have suggested that regional specification is controlled by intrinsic mechanisms (Price and Willshaw 2000). Taking advantage of mice with mutations of the Gbx-2 and Mash-1 genes that have defects in thalamocortical axon projections, recent studies have demonstrated that early regionalization of the neocortex does not require afferent input, instead it is controlled by intrinsic mechanisms (Miya-

[Key Words: Early regionalization; neocortex; COUP-TFI]

${ }^{1}$ Corresponding author.

E-MAIL mtsai@bcm.tmc.edu; FAX (713) 798-8227.

Article and publication are at http://www.genesdev.org/cgi/doi/10.1101/ gad.913601.
shita-Lin et al. 1999; Nakagawa et al. 1999). Therefore, specification and regionalization of the neocortex can be divided into at least two steps: (1) an early regionalization phase in which cortical neurons establish their regional identity through regulating gene expression in a cell-autonomous manner and (2) a refinement phase in which extrinsic influences from subcortical areas, including thalamocortical inputs, shape and maintain the cortical subdivisions. Recent studies showed that neocortical regional identity was shifted in Pax6 and Emx2 mutant mice, indicating that Pax6 and Emx2 are two such intrinsic factors that regulate the regional expression of marker genes and specify cortical identity (Bishop et al. 2000; Mallamaci et al. 2000).

COUP-TFI is an orphan member of the nuclear-receptor superfamily. Mice with a null mutation of the COUP-TFI gene exhibit multiple defects in both central and peripheral nervous systems (Qiu et al. 1997; Zhou et al. 1999). In this study, we have attempted to assess the role of COUP-TFI in early neocortical regionalization. We demonstrated that COUP-TFI is required for the region-specific expression of many marker genes as well as the precise axonal projections between the thalamus and the cortex. Our findings indicate that COUP-TFI is a regulatory factor that works in concert with $\operatorname{Pax} 6$ and $E m x 2$ to specify cortical identity. In addition, COUP-TFI may play a role in maintaining such identity.

\section{Results and Discussion}

Like other regions of the central nervous system, regionalization of the neocortex occurs very early during corticogenesis. It has been proposed that gradients of inductive signals in progenitor cells initiate early neocortical regionalization (Levitt et al. 1997). It also was suggested that the intrinsic factors controlling early regionalization would be expressed in a gradient or in a regionspecific pattern in the developing neocortex (Bishop et al. 2000). Previously, we have shown that COUP-TFI is highly expressed in the developing central nervous system (Qiu et al. 1994). To establish COUP-TFI as a candidate-intrinsic factor for cortical regionalization, we first investigated the expression pattern of COUP-TFI during corticogenesis by section and whole-mount in situ hybridization assays. At E11.5, the onset of cerebral corticogenesis, COUP-TFI expression showed a graded pattern in the neocortex with high-lateral to low-medial expression in the frontal view (Fig. 1A), and high-caudal to low-rostral expression in the sagittal view (Fig. 1B). Section in situ hybridization indicated that the high caudolateral COUP-TFI expression gradient was maintained in the cortical plate even after birth (Fig. 1C,D). This COUP-TFI expression gradient in the superficial layers (layers 2/3) also can be confirmed by whole-mount, in situ hybridization (Fig. 1E,F). Just prior to our submission of this manuscript, the $\mathrm{O}^{\prime}$ Leary laboratory reported a graded expression of COUP-TFI by differential display PCR that is consistent with our findings (Liu et al. 2000). This spatial and temporal expression pattern makes COUP-TFI a likely candidate for a regionalization regulatory factor.

The above finding prompted us to examine the potential role of COUP-TFI in early regionalization by investigating the region-specific expression of marker genes 

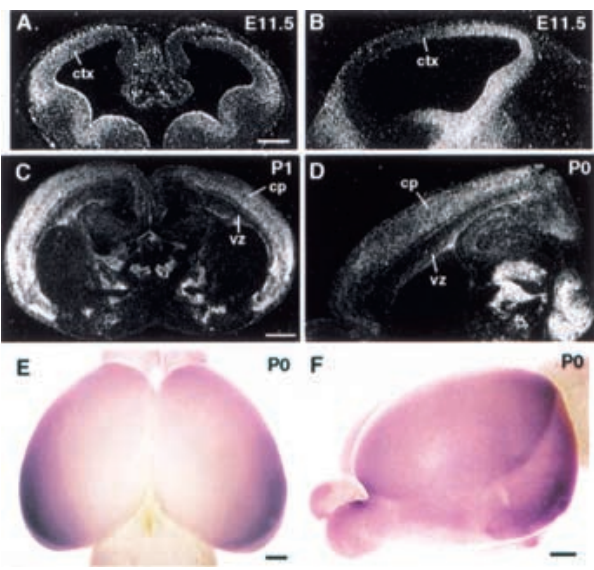

Figure 1. Graded expression of COUP-TFI during corticogenesis. In-situ hybridization using ${ }^{35} \mathrm{~S}$-UTP-labeled COUP-TFI riboprobe on frontal $(A)$ and sagittal $(B)$ sections of E11.5, coronal $(C)$ section of $\mathrm{P} 1$, and sagittal $(D)$ section of $\mathrm{P} 0$ mouse brains. Whole-mount in situ hybridization using digoxigenin-labeled COUP-TFI riboprobe on $\mathrm{PO}$ mouse brains $(E, F)$. Because of limitation of probe penetration, only expression in superficial layers (layers $2 / 3$ ) can be detected by wholemount in situ hybridization. At E11.5, a high-lateral to low-medial and a high-caudal to low-rostral COUP-TFI gradient was observed in frontal $(A)$ and sagittal $(B)$ view. This high-caudolateral expression was detected in the cortical plate after birth as indicated by section $(C, D)$ and whole-mount $(E, F)$ in situ hybridization. cp, Cortical plate; ctx, cortex; vz, ventricular zone. Bar: $A$ and $B, 200 \mu \mathrm{m} ; C-F$, $500 \mu \mathrm{m}$.

including $I d 2, R O R \beta$, and Cadherin 8, which has been used to assess the regionalization of the neocortex in P3/P4 COUP-TFI mutant cortices. Id2, a helix-loop-helix transcription factor, was expressed in a region- and lamina-specific manner (Fig. 2A). Id2 transcripts were detected in the subplate and layers 6,5 , and $2 / 3$, as reported previously (Bulfone et al. 1995). Id2 transcripts can be detected in the upper part of layer 5 and stop abruptly at a rostral boundary (arrow in Fig. 2A) that has been suggested to be between motor and somatosensory cortices (Rubenstein et al. 1999). Id2 expression in layers $2 / 3$ was restricted to the rostral part of the cortex with a boundary within the somatosensory area (arrowhead in Fig. 2A). However, the lamina-specific expression pattern was lost in COUP-TFI mutants (Fig. 2B), which may be because of the greatly reduced number of layer-4 neurons and subplate neurons in COUP-TFI mutants (Zhou et al. 1999) as well as the uniform expression of Id2 in layer 5. More importantly, the rostral boundary in layer 5 that separates motor and sensory cortices was not apparent (Fig. 2B), and Id2 also was expressed throughout the rostro-caudal axis in layers $2 / 3$ in COUP-TFI mutants, suggesting that COUP-TFI is required for regional expression of $I d 2$ in layers 5 and $2 / 3$ neurons.

$R O R \beta$, which encodes an orphan nuclear receptor, is specifically expressed in layer 4 neurons (Park et al. 1997; Schaeren-Wiemers et al. 1997). We have reported previously that $R O R \beta$ expression was greatly reduced at P0 in COUP-TFI mutants because of the death of layer 4 neurons (Zhou et al. 1999). Since the purpose of this investigation was to study the regional expression of $R O R \beta$ rather than compare expression levels, we intentionally took images of $R O R \beta$ transcript in P3 COUPTFI mutants at a much longer exposure time (about twice as long for mutants). Consistent with a previous report (Miyashita-Lin et al. 1999), ROR $\beta$ expression in P3 control-mouse cortex displayed a distinct regional pattern with a caudal boundary within the somatosensory cortex (arrowhead in Fig. 2C) and a region of much reduced expression in a more rostral position (Fig. 2C, between asterisk and arrow). However, in mutant cortex uniform expression of $R O R \beta$ was detected from rostral to caudal cortex with expanded caudal expression (Fig. $2 \mathrm{D})$ indicating that COUP-TFI controls the region-specific expression of $R O R \beta$.

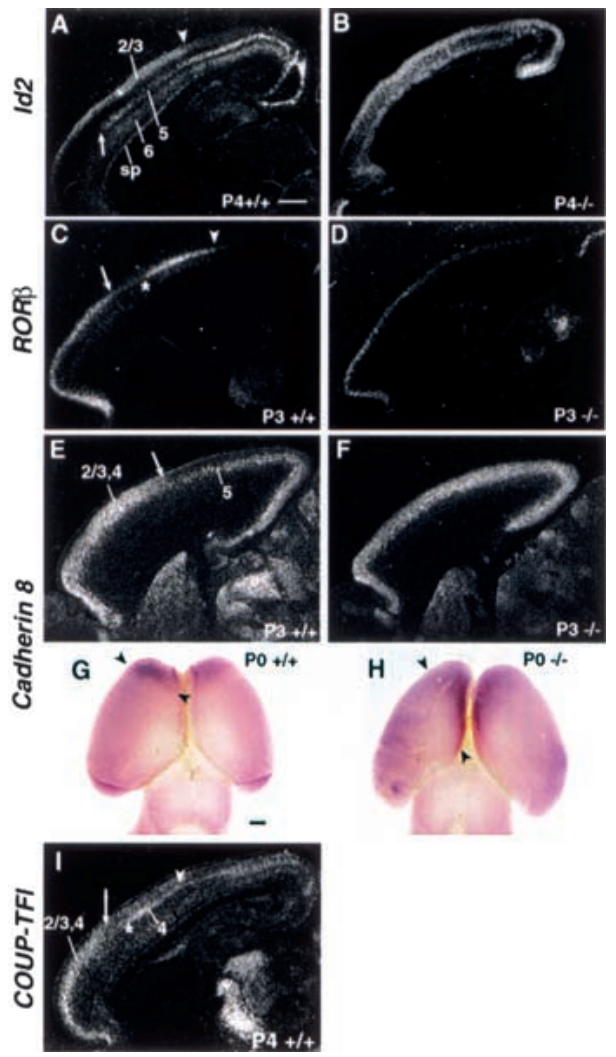

Figure 2. Changes in region-specific expression of $I d 2, R O R \beta$, and Cadherin 8 in COUP-TFI mutants. Section in situ hybridization using Id2 $(A, B), R O R b(C, D)$, Cadherin $8(E, F)$, and COUP-TFI $(I)$ riboprobes on sagittal sections of $\mathrm{P} 3$ or $\mathrm{P} 4$ control $(A, C, E, I)$ and COUP-TFI mutant $(B, D, F)$ cortices. Whole-mount in situ hybridization using digoxigenin-labeled Cadherin 8 riboprobe on P0 control $(G)$ and COUP-TFI mutant $(H)$ brains. Id2 expression in the upper layer of layer 5 stopped abruptly at a rostral boundary (arrow in $A$ ) and in the rostral part of layers $2 / 3$ with a boundary in the somatosensory area (arrowhead in A). However, Id2 expression in P4 COUP-TFI mutant cortex lost its lamina-specific pattern and was uniformly expressed in layers 5 and $2 / 3(B)$. ROR $\beta$ expression in layer-4 neurons showed a caudal boundary (arrowhead in $C$ ) and a region of much-reduced expression in a more rostral position (between arrow and asterisk in $C$ ) in P3 control cortex, while its expression in COUP-TFI mutant was more uniform and expanded caudally with a much lower expression level $(D)$. Cadherin 8 was expressed in the rostral part of the neocortex in the layers $2 / 3$ and 4 (arrow in $E$ ) in P3 control cortex $(E)$. However, in P3 COUP-TFI mutant, Cadherin 8 expression in layers $2 / 3$ and 4 lost its rostral restriction $(F)$. This caudal expansion of Cadherin 8 expression in superficial layers also was observed by whole-mount in situ hybridization (arrowheads in $G, H$ ). In-situ hybridization with COUP-TFI riboprobe revealed that COUP-TFI was expressed throughout the neocortex $(I)$. Specifically, a higher expression in layers $2 / 3$ and 4 in the rostral part (arrow in $I$ ) and a high expression in layer 4 with a boundary in the somatosensory cortex (arrowhead in I) were observed. sp, Subplate. Bar, $500 \mu \mathrm{m}$. 
Cadherin 8 belongs to the type II class of cadherin, a family of cell adhesion molecules that are important for morphogenesis of the central nervous system. At P3, in situ hybridization assay has revealed that Cadherin 8 was uniformly expressed in layer 5 while its expression in layers $2 / 3$ and 4 was restricted to the rostral part of the neocortex (arrow in Fig. 2E). However, in P3 mutant cortex, Cadherin 8 expression in layers $2 / 3$ and 4 lost its rostral restriction and could be detected throughout the rostral-caudal axis, while expression in layer 5 was unchanged (Fig. 2F). The expression of Cadherin 8 in superficial layers (layers 2/3) also was detected by wholemount, in situ hybridization (Fig. 2G,H). Cadherin 8 was expressed in the frontal area of the neocortex with a boundary around the limit of motor cortex (arrowheads in Fig. 2G) in P0 control brains. The rostral Cadherin 8 expression boundary in superficial layers was shifted caudally, especially in the medial region, in P0 mutant cortices (arrowheads in Fig. 2H). Therefore, the loss of restricted rostral expression of Cadherin 8 in layers $2 / 3$ and 4 indicated that COUP-TFI is required for regionspecific expression of Cadherin 8. Taken together, the region-specific expression of many genes including $I d 2$, $R O R \beta$, and Cadherin 8 around $\mathrm{P} 4$, when cortical lamination is close to completion, is altered in COUP-TFI mutants indicating that normal neocortical regionalization requires COUP-TFI. Since expression of these marker genes is regulated by intrinsic mechanisms and is independent of thalamocortical innervation (Miyashita-Lin et al. 1999; Nakagawa et al. 1999), our findings establish COUP-TFI as one of the intrinsic regulatory factors that control the specification of the neocortex.

Interestingly, COUP-TFI itself also was expressed in a region-specific manner at $\mathrm{P} 4$ (Fig. 2I). Although COUPTFI transcripts were detected throughout the cortex, a higher expression in the rostral part of layers $2 / 3$ and 4 (arrow in Fig. 2I) was observed and it correlated with Cadherin 8 expression in layers $2 / 3$ and 4 . In addition, COUP-TFI was highly expressed in layer 4 neurons (Fig. 2I) with several boundaries (arrow, asterisk, and arrowhead in Fig. 2I) that resembled the expression pattern of ROR $\beta$ (Fig. 2C). Although the expression pattern of COUP-TFI in the postnatal cortex correlates with components of some marker genes, it differs considerably from other expression patterns, suggesting that COUPTFI is important for maintaining region-specific gene expression of some but not all of the markers examined.

Because expression of COUP-TFI displayed a regionspecific pattern very early (we can detect it at E11.5) during corticogenesis. We also investigated the expression patterns of $I d 2, R O R \beta$, and Cadherin 8 in late embryonic development. We chose E17.5 because this is a time when generation of cortical plate neurons has been completed (around early E17) (Caviness et al. 1995) and massive innervation of the cortical plate by thalamic axons has not yet started. However, at this stage, most cortical plate neurons still are migrating along radial glia, so layer-specific expression cannot be identified. Id2 expression displayed a high-caudal to low-rostral gradient in control cortex, including the cortical plate and the ventricular zone. Its expression in mutants, though, showed a uniform rostral-to-caudal expression throughout the cortex (Fig. 3A,B). ROR $\beta$ expression in control cortex showed a high-rostral to low-caudal gradient in the cortical plate (layer 4 neurons), which was interrupted in the future somatosensory cortex in control

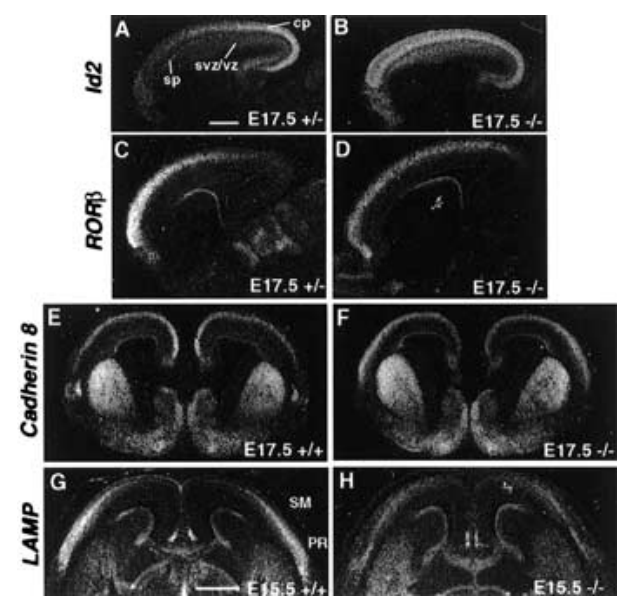

Figure 3. Altered expression of $I d 2, R O R \beta$, Cadherin 8 , and LAMP during embryonic development. Section in situ hybridization using $\operatorname{Id} 2(A, B), R O R \beta(C, D)$, Cadherin $8(E, F)$, and $L A M P(G, H)$ riboprobes on sagittal $(A-D)$ and coronal $(E-H)$ sections of E17.5 or E15.5 control $(A, C, E, G)$ and COUP-TFI mutant $(B, D, F, H)$ cortices. Expression of Id2 exhibited a high-caudal to low-rostral gradient at E17.5 in control cortex $(A)$, while it was uniformly expressed in COUP-TFI mutant $(B)$. The high-rostral to low-caudal $R O R \beta$ expression gradient in E17.5 control cortex $(C)$ also became more uniform in COUPTFI mutants $(D)$. The high-medial to low-lateral Cadherin 8 expression $(E)$ in control cortices was switched to high-lateral to lowmedial in COUP-TFI mutants (F). LAMP transcripts were detected in the cortex of control E15.5 mice with a high-lateral to low-medial gradient $(G)$. However, in COUP-TFI mutant mice, LAMP expression level in the cortex was greatly reduced with a rather uniform expression and an extended medial boundary $(H)$. cp, Cortical plate; $\mathrm{PR}$, the perirhinal cortex; SM, the sensorimotor cortex; sp, subplate; svz, subventricular zone; vz, ventricular zone. Bar, $500 \mu \mathrm{m}$.

E17.5 cortex (Fig. 3C). Mutation of COUP-TFI resulted in the uniform expression of $R O R \beta$, and in it having a more caudal boundary (Fig. 3D). Expression of Cadherin 8 in control cortical plate showed a high-medial to lowlateral pattern, while its transcripts displayed a high-lateral to low-medial pattern in the mutant cortices (Fig. $3 \mathrm{E}, \mathrm{F})$. Taken together, COUP-TFI regulates region-specific expression of Id2, ROR $\beta$, and Cadherin 8 before E17.5. The fact that graded or region-specific expression of these marker genes was lost at E17.5 (Fig. 3), a time before thalamic axons innervate the cortical plate, again confirms that early regionalization of the neocortex is independent of thalamic innervation. Therefore, specification of the neocortex by COUP-TFI occurs shortly after the fate of cortical plate neurons is determined and before the innervation of the cortical plate by thalamic axons takes place.

To further demonstrate that COUP-TFI regulates region-specific gene expression independent of thalamic influence, we investigated the expression of other earlier markers, which are expressed before the thalamocortical axons ever reach the cortex. The limbic system-associated membrane protein (LAMP) is one of the earliest markers of cortical specification (Horton and Levitt 1988). In cerebral cortex, $L A M P$ is expressed in early differentiated neurons in the limbic cortical region. At E15.5, a high-lateral to low-medial expression gradient, with the highest signal in the perirhinal cortex and a gradually decreased signal towards the sensorimotor cortex, was detected in control mice as previously reported (Fig. 3G) (Pimenta et al. 1996). However, in COUP-TFI 
mutant mice, $L A M P$ expression level in the cortex was greatly reduced. More importantly, the high-lateral to low-medial $L A M P$ expression gradient was not obvious. Instead, $L A M P$ was expressed in a rather uniform manner from the perirhinal cortex to the sensorimotor cortex with an extended medial boundary (Fig. $3 \mathrm{H}$ ). These results clearly demonstrated that COUP-TFI is an intrinsic factor for early cortical regionalization, which plays an important role in specification of the cerebral cortex since the onset of the corticogenesis.

During the processing of sensory information, various functionally distinct regions in the neocortex communicate with corresponding areas in the thalamus by way of precise synaptic connections. These connections between the cortex and the thalamus are reciprocal and in a region-specific manner. For example, the somatosensory cortex receives afferent inputs from, and sends efferent projections to, the ventrobasal thalamus (VB) while the visual cortex connects with the lateral genuculate nucleus (LGN). To test the functional consequences of the altered neocortical regionalization in COUP-TFI null mice, we investigated the precise connections between the cortex and the thalamus by dyetracing experiments. Lipophilic DiI and DiA crystals were placed in the somatosensory and visual cortices respectively (see Fig. 4A, $\mathrm{A}^{\prime}$ for positions). Two types of terminal ends were visualized in the terminal fields in the thalamus (Fig. 4B). Those large ends are retrogradely labeled thalamic neurons while those small ends are boutons, the axonal terminals of cortical neurons. Therefore, the labeling we observed in the thalamus actually is the result of the sum of both retrogradely labeled thalamocortical and anterogradely labeled corticothalamic projections. As expected, placing DiI crystals in the somatosensory cortex of the control brains resulted in DiI labeling in the VB (Fig. 4D, red) while placing DiA in the visual cortex labeled the LGN (Fig. 4D, green). When similar experiments were performed in COUP-TFI mutant brains, we first observed a greatly reduced DiI labeling in the VB (Fig. 4F, red), which is consistent with our previously reported, misguided thalamocortical projections in COUP-TFI mutants. Placing DiI in the mutant somatosensory cortex labeled the VB as it did in the control brains (Fig. 4F, red). However, placing DiA crystals in the presumptive visual cortex position led to DiA labeling in the VB, which normally is connected with the somatosensory cortex, rather than its usual target the LGN (Fig. 4F, green). Because the development of the thalamus was normal in P0 mutants as indicated by the normal expression of several thalamic-marker genes such as Gbx2, Id4, and Lef1 (data not shown), such miswired corticothalamic connections suggested that the cortical area, which usually is within the visual cortex, adopted the identity of the somatosensory cortex in terms of neural connectivity in mice lacking COUP-TFI. Thus, these results confirm that COUP-TFI is required for the proper regionalization of the cortex.

Recently, it has been reported that the intrinsic factors, Pax6 and Emx2 also regulate the region-specific expression of Cadherin 6 and Cadherin 8 (Bishop et al. 2000). To elucidate the possible link between COUP-TFI and $P a x 6 / E m \times 2$, we investigated the graded expression of Pax6 and Emx2 in COUP-TFI mutants. In a pattern similar to that observed in the control cortices, Pax6 transcripts were detected in a high-rostral to low-caudal gradient in sagittal sections (Fig. 5A,B) and in a high-

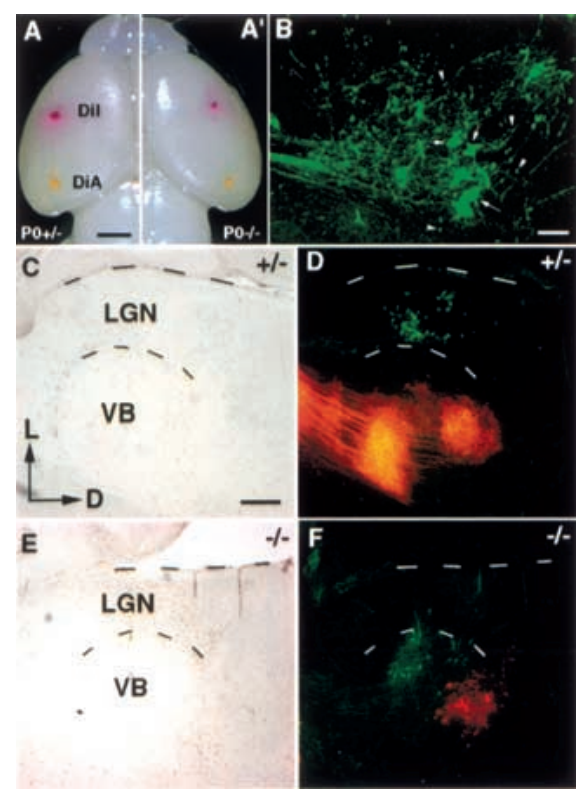

Figure 4. Changes in region-specific connections between the cortex and the thalamus in COUP-TFI mutants. $\left(A, A^{\prime}\right)$ Shows the positions of DiI and DiA crystals, which were placed in the somatosensory and the visual cortices, respectively. When visualized in the coronal sections of the thalamus using a laser-scanning confocal microscope $(B)$, two types of labeling were observed: the large ends were retrogradely labeled thalamic neurons (arrows in $B$ ) and the small ends were boutons of anterogradely labeled axonal terminals of cortical neurons (arrowheads in $B$ ). $C, D$ and $E, F$ were conventional microscope images. $C$ and $E$ were bright fields. $D$ and $F$ were overlapping fluorescent images with Texas Red (for DiI labeling, red) and FITC (for DiA labeling, green) filters. Placing DiI and DiA crystals in control somatosensory or visual cortices resulted in labeling in the VB and the LGN, respectively (red and green in $D$ ). Consistent with our previous report of defects in thalamocortical projections, insertion of DiI in the somatosensory cortex of COUP-TFI mutants resulted in a much less labeling in the thalamus (red in F). Placing DiI in somatosensory cortex led to labeling in the VB as it did in the controls (red in F). However, when DiA crystals were placed in the presumptive visual cortex of COUP-TFI mutants, most of labeling was seen in the VB instead of the LGN (green in F). D, Dorsal; L, lateral; LGN, lateral geniculate nucleus; VB, ventrobasal thalamus. Bar: $A, 1 \mathrm{~mm} ; B, 50 \mu \mathrm{m} ; C, 200 \mu \mathrm{m}$.

lateral to low-medial gradient in coronal sections (Fig. 5C,D) in E13.5 mutant cortices. The gradient expression of Emx2, which normally was in a high-caudal to lowrostral (Fig. 5E,F) and high-medial to low-lateral (Fig. $5 \mathrm{G}, \mathrm{H})$ pattern, also was maintained in COUP-TFI mutant cortices at E13.5. The unchanged, graded expression of Pax6 and Emx2 in COUP-TFI mutant cortices indicated that COUP-TFI, Pax6, and Emx2 might function independently in regulating early regionalization of the neocortex. In addition, the fact that COUP-TFI is expressed not only in the ventricular zone but also in the cortical plate, while Pax6 and Emx2 are expressed mainly in the ventricular zone with complementary expression gradients suggests that COUP-TFI does not act downstream of Pax6 or Emx2 in the cortical neurons. Additionally, the difference in altered expression profiles (e.g., Cadherin 8) between COUP-TFI null mice and Pax6 and Emx2 mutants further suggests that COUP. TFI and Pax6/Emx2 act through different pathways and these intrinsic factors work in concert to establish the identity of each subdivision of the neocortex. 


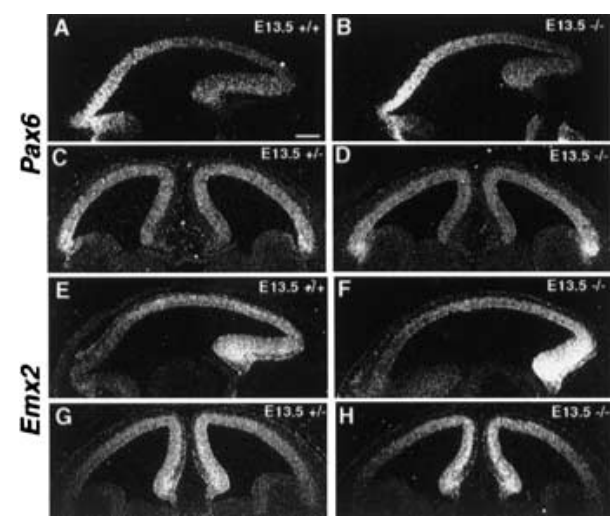

Figure 5. Normal-graded expression of $\mathrm{Pax} 6$ and $E m \times 2$ in the cortices of E13.5 COUP-TFI mutants. Section in situ hybridization using Pax6 $(A-D)$ and Emx2 $(E-H)$ riboprobes on sagittal $(A, B, E, F)$ and frontal $(C, D, G, H)$ E13.5 control $(A, C, E, G)$ and COUP-TFI mutant $(B, D, F, H)$ cortices. Pax 6 was expressed in a high-rostral to lowcaudal $(A, B)$ and high-lateral to low-medial $(C, D)$ pattern in control as well as in mutant cortices. Meanwhile, expression of $E m \times 2$ was in a high-caudal to low-rostral $(E, F)$ and high-medial to low-lateral $(G, H)$ pattern in control as well as in mutant cortices. Bar, $200 \mu \mathrm{m}$.

While the graded expression of Pax6 and Emx2 is lost around E15.5 and E16.5, respectively (Walther and Gruss 1991; Gulisano et al. 1996), the graded COUP-TFI expression was maintained in the neocortex even after birth (Fig. 1C-F). Furthermore, COUP-TFI transcript can be detected throughout the cortex during corticogenesis while Pax6 and Emx2 are mainly confined to the ventricular zone (Walther and Gruss 1991; Gulisano et al. 1996; Mallamaci et al. 1998). In addition, COUP-TFI expression at P4 also was region-specific (Fig. 2I) and certain domains correlated well with those of Cadherin 8 and $R O R \beta$. These findings suggest that COUP-TFI is not only required for initiating the early regionalization of the neocortex but also may play a role in maintaining such regional identity, while Pax6 and Emx2 mainly function in initiation of the early regionalization.

The molecular mechanisms behind how intrinsic factors such as COUP-TFI, Pax6, and Emx2 regulate regionspecific gene expression still is largely unknown. We do not know if intrinsic factors act directly or indirectly in regulating transcription of marker genes. The complexity and diversity of the expression patterns suggest that early regional specification is achieved through the cooperation of multiple intrinsic factors. As a transcription factor, COUP-TFI regulates gene transcription through both transrepression and transactivation (Zhou et al. 2000) mechanisms. It will be interesting to determine how COUP-TFI controls the region-specific expression of those marker genes. Moreover, understanding the mechanism whereby the graded expression of COUPTFI, Pax6, and Emx2 is established will greatly enhance our understanding of how neocortical regionalization takes place.

Previously, we reported defects in guidance of thalamocortical projections in COUP-TFI mutants (Zhou et al. 1999). We proposed that improper differentiation of subplate neurons in COUP-TFI null mice may lead to altered expression of axon guidance molecules by these subplate neurons and, consequently, impaired thalamocortical projections. These guidance molecules can be diffusible molecules, membrane proteins, membrane- bound molecules, or extracellular-matrix molecules. Increasing evidence suggests that the cortex also may produce such guidance molecules (Price and Willshaw 2000) to guide thalamocortical axons to their proper targets by either promoting or inhibiting outgrowth of these axons. In this study, we demonstrated that the specification of the cortical areas in COUP-TFI mutants was compromised and it is possible this defect altered expression of such guidance molecules in the cortex. As a matter of fact, expression of one such molecule, $L A M P$, was changed in COUP-TFI mutants. However, in COUP-TFI mutants, most thalamocortical axons lost their direction as soon as they left the internal capsule. Because the distance from the internal capsule to the cortex may be beyond the range that diffusible signals can function, and because thalamocortical axons leave the internal capsule before the majority of cortical-plate neurons are generated, defects in the mutant cortex (other than the subplate) are unlikely the main reason for misguidance of thalamocortical axons at the internal capsule.

\section{Material and methods}

Mice

COUP-TFI mouse breeding and husbandry was as described (Zhou et al. 1999). For this study, four pair of mice ranging from P3 to P4 were collected for section in situ hybridization. More than 10 pairs of $\mathrm{P} 0$ control and mutant mouse brains were used for whole-mount in situ hybridization experiments with COUP-TFI and Cadherin 8 probes. Five pairs of PO brains were used for DiI/DiA labeling experiments.

\section{Section in situ hybridization}

Section in situ hybridization was performed as described previously (Zhou et al. 1999). The cDNA templates were $1.5 \mathrm{~kb}$ of COUP-TFI cDNA, $1.9 \mathrm{~kb}$ of ROR $\beta$ cDNA (Dr. Michael Becker-Andre, Glaxo Wellcome Research and Development S.A., Switzerland), 235 bp of 3'-UTR of Id2 cDNA (Dr. Mark Israel, University of California, San Francisco), 1.1 $\mathrm{kb}$ of Cadherin $8 \mathrm{cDNA}$ (Dr. Masatoshi Takeichi, Kyoto University, Japan), 260 bp of Pax6 cDNA (Dr. Peter Gruss, Max Planck Institute for Biophysical Chemistry, Germany), 300 bp of Emx2 cDNA (Dr. Shinichi Aizawa, Kumamoto University School of Medicine, Japan), and 442 bp LAMP cDNA (+50-+491, generated by RT-PCR).

Whole-mount in situ hybridization

Whole-mount in situ hybridization was carried out using digoxigeninlabeled antisense cRNA probes as described previously (Qiu et al. 1997). Due to limitation of probe penetration, only expression in superficial layers (layers 2/3) can be detected by whole-mount in situ hybridization.

\section{DiI and DiA labeling}

Brains from newborn mice (PO) were removed and fixed in $4 \%$ buffered paraformaldehyde. Crystals of fluorescent dyes DiI and DiA (Molecular Probes) were inserted into the primary somatosensory or visual cortices, respectively (see Fig. 4A and A' for positions). Brains were incubated in fixative at room temperature for $10 \mathrm{wk}$, embedded in $4 \%$ low-meltingpoint agarose, and sectioned in the coronal plane at $125 \mu \mathrm{m}$ using a Leica VT1000S vibratome. Axonal terminals in the thalamus were visualized using a conventional fluorescence microscope (Zeiss Axiophot) and a laser-scanning confocal microscope (Zeiss LSM 510).

\section{Acknowledgments}

We thank Drs. Michael C. Crair and Austin Cooney for their critique of this manuscript. We are grateful to Drs. Mark Israel (Id2), Masatoshi Takeichi (Cadherin 8), Michael Becker-Andre (ROR $\beta)$, Peter Gruss (Pax6), and Shinichi Aizawa (Emx2) for cDNAs. We thank Jing Xu and Meijin Chu for excellent technical assistance. C.Z. is supported by U.S. Public Research Service Award Fellowship Awards. This work is supported by grants from the National Institute of Health to M.J.T. and S.Y.T. 
The publication costs of this article were defrayed in part by payment of page charges. This article must therefore be hereby marked "advertisement" in accordance with 18 USC section 1734 solely to indicate this fact.

\section{References}

Bishop, K.M., Goudreau, G., and O'Leary, D.D. 2000. Regulation of area identity in the mammalian neocortex by Emx2 and Pax6. Science 288: 344-349.

Bulfone, A., Smiga, S.M., Shimamura, K., Peterson, A., Puelles, L., and Rubenstein, J.L. 1995. T-brain-1: A homolog of Brachyury whose expression defines molecularly distinct domains within the cerebral cortex. Neuron 15: 63-78.

Caviness, V.S., Takahashi, T., and Nowakowski, R.S. 1995. Numbers, time and neocortical neuronogenesis: A general developmental and evolutionary model. Trends Neurosci. 18: 379-383.

Gulisano, M., Broccoli, V., Pardini, C., and Boncinelli, E. 1996. Emx1 and Emx2 show different patterns of expression during proliferation and differentiation of the developing cerebral cortex in the mouse. Eur. J. Neurosci. 8: 1037-1050.

Horton, H.L. and Levitt, P. 1988. A unique membrane protein is ex pressed on early developing limbic system axons and cortical targets. I. Neurosci. 8: 4653-4661.

Levitt, P., Barbe, M.F., and Eagleson, K.L. 1997. Patterning and specification of the cerebral cortex. Annu. Rev. Neurosci. 20: 1-24.

Liu, Q., Dwyer, N.D., and O'Leary, D.D. 2000. Differential expression of COUP-TFI, CHL1, and two novel genes in developing neocortex identified by differential display PCR. J. Neurosci. 20: 7682-7690.

Mallamaci, A., Iannone, R., Briata, P., Pintonello, L., Mercurio, S., Boncinelli, E., and Corte, G. 1998. EMX2 protein in the developing mouse brain and olfactory area. Mech. Dev. 77: 165-172.

Mallamaci, A., Muzio, L., Chan, C.H., Parnavelas, J., and Boncinelli, E. 2000. Area identity shifts in the early cerebral cortex of Emx2-/mutant mice. Nat. Neurosci. 3: 679-686.

Miyashita-Lin, E.M., Hevner, R., Wassarman, K.M., Martinez, S., and Rubenstein, J.L. 1999. Early neocortical regionalization in the absence of thalamic innervation. Science 285: 906-909.

Nakagawa, Y., Johnson, J.E., and O'Leary, D.D. 1999. Graded and areal expression patterns of regulatory genes and cadherins in embryonic neocortex independent of thalamocortical input. I. Neurosci. 19: 10877-10885.

O'Leary, D.D. 1989. Do cortical areas emerge from a protocortex? Trends Neurosci. 12: 400-406.

Park, H.T., Kim, Y.J., Yoon, S., Kim, J.B., and Kim, J.J. 1997. Distributional characteristics of the mRNA for retinoid $\mathrm{Z}$ receptor beta $(\mathrm{RZR}$ beta), a putative nuclear melatonin receptor, in the rat brain and spinal cord. Brain Res. 747: 332-337.

Pimenta, A.F., Reinoso, B.S., and Levitt, P. 1996. Expression of the mRNAs encoding the limbic system-associated membrane protein (LAMP): II. Fetal rat brain. J. Comp. Neurol. 375: 289-302.

Price, D.J. and Willshaw, D.J. 2000. Mechanisms of cortical development. Oxford University Press, Oxford, UK.

Qiu, Y., Cooney, A.J., Kuratani, S., DeMayo, F.J., Tsai, S.Y., and Tsai, M.J. 1994. Spatiotemporal expression patterns of chicken ovalbumin upstream promoter-transcription factors in the developing mouse central nervous system: evidence for a role in segmental patterning of the diencephalon. Proc. Natl. Acad. Sci. 91: 4451-4455.

Qiu, Y., Pereira, F.A., DeMayo, F.J., Lydon, J.P., Tsai, S.Y., and Tsai, M.J. 1997. Null mutation of mCOUP-TFI results in defects in morphogenesis of the glossopharyngeal ganglion, axonal projection, and arborization. Genes \& Dev. 11: 1925-1937.

Rakic, P. 1988. Specification of cerebral cortical areas. Science 241: 170176.

Rubenstein, J.L., Anderson, S., Shi, L., Miyashita-Lin, E., Bulfone, A., and Hevner, R. 1999. Genetic control of cortical regionalization and connectivity. Cereb. Cortex 9: 524-532.

Schaeren-Wiemers, N., Andre, E., Kapfhammer, J.P., and Becker-Andre, M. 1997. The expression pattern of the orphan nuclear receptor ROR $\beta$ in the developing and adult rat nervous system suggests a role in the processing of sensory information and in circadian rhythm. Eur. J. Neurosci. 9: 2687-2701.

Walther, C. and Gruss, P. 1991. Pax-6, a murine paired box gene, is expressed in the developing CNS. Development 113: 1435-1449.

Zhou, C., Qiu, Y., Pereira, F.A., Crair, M.C., Tsai, S.Y., and Tsai, M.J 1999. The nuclear orphan receptor COUP-TFI is required for differ entiation of subplate neurons and guidance of thalamocortical axons. Neuron 24: 847-859.

Zhou, C., Tsai, S.Y., and Tsai, M. 2000. From apoptosis to angiogenesis New insights into the roles of nuclear orphan receptors, chicken ovalbumin upstream promoter-transcription factors, during development. Biochem. Biophys. Acta. 1470: M63-M68. 


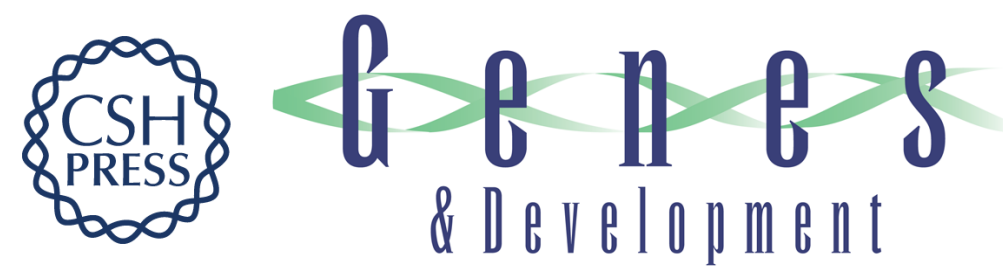

\section{COUP-TFI: an intrinsic factor for early regionalization of the neocortex}

Cheng Zhou, Sophia Y. Tsai and Ming-Jer Tsai

Genes Dev. 2001, 15:

Access the most recent version at doi:10.1101/gad.913601

References This article cites 22 articles, 9 of which can be accessed free at: http://genesdev.cshlp.org/content/15/16/2054.full.html\#ref-list-1

License

Email Alerting Receive free email alerts when new articles cite this article - sign up in the box at the top Service right corner of the article or click here.

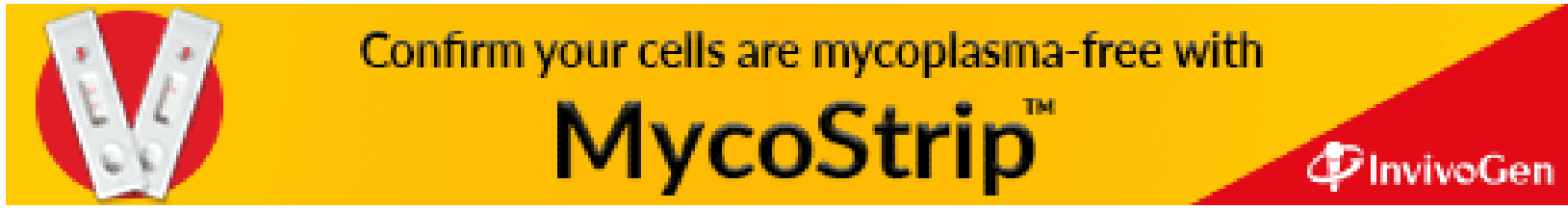

